\title{
CHEMICO-PHYSICAL MODELS OF COMETARY ATMOSPHERES
}

\author{
W. F. Huebner, J. J. Keady, and D. C. Boice \\ T-4 \\ Los Alamos National Laboratory \\ Los Alamos, NM 87545 \\ USA
}

H. U. Schmidt and R. Wegmann

Max-Planck-Institut für Astrophysik

Kar1-Schwarzschild-Straße 1

D-8046 Garching

Fed. Rep. Germany

\begin{abstract}
Sublimation (vaporization) of the icy component of a cometary nucleus determines the initial composition of the coma gas as it streams outward and escapes. Photolytic reactions in the inner coma, escape of fast, light species such as atomic and molecular hydrogen, and solar wind interaction in the outer coma alter the chemical composition and the physical nature of the coma gas. Models that describe these interactions must include (1) chemical kinetics, (2) coma energy balance, (3) multifluid flow for the rapidly escaping light components, the heavier bulk fluid, and the plasma with separate temperatures for electrons and the remainder of the gas, (4) transition from a collision dominated inner region to free molecular flow of neutrals in the outer region, (5) pickup of cometary ions by the solar wind, (6) counter and cross streaming of neutrals with respect to the plasma which outside of the contact surface also contains solar wind ions, and (7) magnetic fields carried by the solar wind. Progress on such models is described and results including velocity, temperature, and number density profiles for important chemical species are presented and compared with observations.
\end{abstract}

\section{INTRODUCTION}

Astrochemistry of comets has its beginning at the time and location of the origin of cometesimals. The place and time is still being debated; various possibilities for their origin include the intermediate or outer solar nebula during the early stages of formation of the solar system, the presolar nebula, or a companion cloud to the presolar nebula. In the latter two cases interstellar molecules play an important role in the composition of comets. We will not discuss the origin of comets nor the chemico-physical changes that they undergo while in "storage" in the 
Oort cloud. But it is clear that astrochemistry must eventually lead to a consistent picture from the birth of comets at their place of origin to their death in the inner solar system.

In section 2 we discuss the chemico-physical processes occurring in the inner coma of a comet in the vicinity of the sun. Rather than give a complete description of all processes, we only summarize those that have already been published and concentrate on new developments such as the energy budget for the electrons, which results in a region of hot electrons with temperature much higher than the gas temperature. The effect of these hot electrons is to enhance dissociation and ionization through collisions, which are about as important as the corresponding photoprocesses in this region.

In section 3 we discuss the coma - solar wind interaction. For this region we model counter and cross streaming of a plasma with coma neutrals in an axisymmetric calculation. This model determines the bow shock, contact surface, and inner shock together with the chemical composition, but does not yet include the dynamic effects of the magnetic field. The neutral coma molecules stream radially outward, virtually unimpeded by the contact surface. The plasma is treated as a fluid containing coma ions inside the contact surface and a mixture of coma ions and solar wind ions outside the contact surface. Solar wind interaction affects mainly the ion distribution. Inside the contact surface ions flowing towards the sun are turned around to flow towards the tail. This velocity change alters their density distribution. Outside of the contact surface ions are created by the same processes as inside of it, but ionization is further enhanced by the solar wind through charge exchange reactions and electron collisions. Additional hot electrons can be generated by the Alfuén critical velocity phenomenon. The ions are picked up by the solar wind, mass loading it and slowing it down. The self-consistently calculated composition is then represented by a mean ion mass and velocity and a mean neutral mass and velocity in a threedimensional calculation that also contains the magnetic field. This provides an internally consistent interpretation of a comet, from the solar wind interaction region to the nuclear surface.

Section 4 contains results from our model and makes comparisons to observations and to data obtained from the ICE spacecraft encounter with comet Giacobini - Zinner.

\section{THE INNER COMA}

The inner coma is the region between the nucleus and the contact surface. Energy balance at the nuclear surface between insolation (mostly in the visible spectrum), reflection as determined from the albedo, reradiation in the infrared, and absorption by the surface layer, coupled to equations of state for the sublimating (evaporating) gas determines the vapor density of the mother molecules, the gas temperature, and the sound speed of the gas (see, e.g., Huebner, 1985). The equations of 
state that we use are the clausius - clapeyron equation for the solid gas interface and the ideal gas law for the gas. The latent heat of sublimation is determined by the assumed composition of the ice component on the nuclear surface. The albedo and infrared emissivity are parameters. We have assumed that the icy component of the nucleus is a mixture of $\mathrm{H}_{2} \mathrm{O}, \mathrm{CO}_{2}, \mathrm{NH}_{3}, \mathrm{CH}_{4}, \mathrm{CO}, \mathrm{H}_{2} \mathrm{CO}, \mathrm{HCOOH}, \mathrm{CH}_{3} \mathrm{OH}, \mathrm{HCN}, \mathrm{CH}_{3} \mathrm{CN}, \mathrm{N}_{2}$, $\mathrm{CH}_{3} \mathrm{NH}_{2}, \mathrm{H}_{2} \mathrm{C}_{3} \mathrm{H}_{2}$, and $\mathrm{C}_{2} \mathrm{H}_{2}$. Sôlar ultraviolet photodestruction of the ${ }^{2}$ mother molecules and important daughter species are calculated with the effects of detailed wavelength-dependent optical depth included. An extensive set of new solar photo rate coefficients have been calculated. A one-dimensional, time-dependent calculation (assuming expansion and radiative cooling, chemical and dissociative heating, and fast flowing atomic and molecular hydrogen) solves the coupled rate equations and predicts density, pressure, temperature, and velocity profiles, and abundances of the chemical species. The visible sunlight causes the gas to sublimate from the nucleus, while the primary source for the dissociation and ionization of the mother molecules is ultraviolet sunlight. This heats the coma gas and the electrons and creates radicals and ions that are chemically reactive. Infrared radiation is an important cooling process in the coma. An escape probability for fast hydrogen (Huebner and Keady, 1984) has been calculated specifically for coma density profiles and has been incorporated into the comet model program.

The excess energy $E_{x}$ in photodissociation, photoionization, and photodissociative ionization is defined by

$E_{x}=\int_{0}^{\lambda_{t h}} \operatorname{hc}\left(\frac{1}{\lambda}-\frac{1}{\lambda_{t h}}\right) \sigma(\lambda) F(\lambda) e^{-T(\lambda)} \mathrm{d} \lambda / \int_{0}^{\lambda_{\text {th }}} \sigma(\lambda) F(\lambda) \mathrm{e}^{-\tau(\lambda)} \mathrm{d} \lambda$

Where $F(\lambda) d \lambda$ is the wavelength dependent solar flux (number of photons per unit area per unit time between wavelength $\lambda$ and $\lambda+d \lambda), \sigma(\lambda)$ is the photo cross section as a function of wavelength, and $T(\lambda)$ is the wavelength-dependent optical depth of the coma gas. In photodissociation most of the excess energy goes into translational motion of the lightest product because of momentum conservation. Since dissociation products are created with velocities in random directions with respect to the outstreaming gas, this excess energy manifests itself as heat. Typically the excess energy for dissociation is a $\mathrm{few} \mathrm{eV}$ to about $10 \mathrm{eV}$.

For photoionizations and photodissociative ionizations practically all of the excess energy goes into heating of electrons, since they are lighter than the other photofragments by at least a factor of 1820 . The excess energy in photoionization is about $10 \mathrm{eV}$ and in dissociative ionization it is several tens of electron volts. Since the photo cross sections in general decrease with decreasing wavelength, photons with the shortest wavelength can penetrate deepest into the coma and thus excess energies in the innermost coma can be about $100 \mathrm{eV}$. Because only very few of these photoprocesses occur (the cross sections are small) and the gas density is high, these photoproducts equilibrate rapidly and their heating effect is very small. 
The gas cools as it expands, but it also becomes optically thinner and soon the heating of the bulk gas and the electrons dominates over the cooling effects. The electrons thermalize rapidly by collisions with polar molecules, primarily water. However, at some point the electrons thermally decouple from the coma gas and their temperature, T ' $^{3}$ rises suddenly and rapidly as shown in Fig. 1. This occurs at $R \simeq \mathbf{1 0}^{3}$ $\mathrm{km}$ from the nucleus and is not very sen-

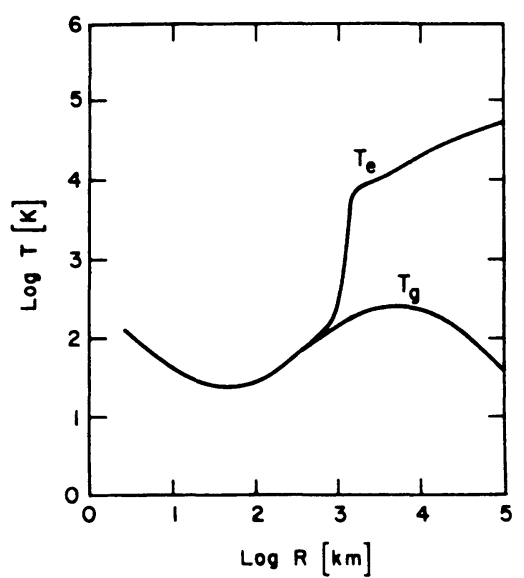

Figure 1. Electron temperature, $\mathrm{T}_{\mathrm{e}}$, and gas temperature, $\mathrm{T}_{\mathrm{g}}$, vs. $\mathrm{distance}$ in the coma. sitive to chemical composition or heliocentric distance $r$ (at least not for $r<$ $1.6 \mathrm{AU})$. Similar results were obtained by Marconi and Mendis (1984). These hot electrons can cause secondary, collisional dissociation, ionization, and dissociative ionization. However, dissociative electron recombination decreases with increasing electron temperature typically like $\mathrm{T}^{-192}$. The net effect is that production of molecular radicals and ions is increased over that from photoprocesses alone by about a factor of two. Number densities for neutrals have been plotted in Fig. $2 a$ and separately for ions in $F i g .2 b$ as a function of cometocentric distance for an assumed mixture containing $43 \% \mathrm{H}_{2} \mathrm{O}$, $22 \% \mathrm{H}_{2} \mathrm{CO}, 13 \% \mathrm{CH}_{4}, 12 \% \mathrm{CO}_{2}, 5 \% \mathrm{~N}_{2}, \quad 3 \%$ $\mathrm{CO}$, and trace amounts of the other molecules mentioned above. Collisional effects caused by the hot electrons at $R$ $\approx 10^{3} \mathrm{~km}$ are particularly noticable in Fig. $2 \mathrm{~b}$, but the resultant increase in chemical reactions adds to the complexity of the analysis.

\section{THE INTERACTION REGION}

As discussed, e.g., by Schmidt and Wegmann (1982), a contact surface separates purely cometary plasma from the mass loaded solar wind plasma. The solar wind interacts strongly with the cometary ions, but only little with the neutrals. Therefore the neutrals continue to expand radially outside the contact surface where they are progressively ionized, removed from the neutral flow, and added to the plasma flow. This provides an extended source of ions which disturbs the solar wind in a region determined by the ionization life time of the neutrals. The solar wind carries an embedded magnetic field that imposes a common velocity on all ions, at least in the direction normal to the field. Thus the ions are "picked up" by the solar wind plasma as soon as they are born, starting in the forefield outside of the bow shock. The unperturbed solar wind is a hypersonic parallel flow with a velocity of about $400 \mathrm{~km} / \mathrm{s}$ and a density of about 5 protons and 5 electrons $\mathrm{cm}^{-5}$ that is gradually loaded with the newly born, slow, and massive ions that decelerate it and heat it. The flow can remain parallel and super- 
sonic only as long as the mass density of the added ions is less than one third of the mass density of the solar wind. When this value is exceeded a transition through a shock front causes the flow to become subsonic and divergent. Since the Mach number was already decreased in the forefield, the shock will be weak. In our macroscopic fluid model, the shock appears as a sharp discontinuity of hyperbolic shape which is axisymmetric with respect to the central streamline through the stagnation point. In reality the shock profile must extend over more than one gyration radius of a cometary pickup ion. The distance of the bow shock from the nucleus is determined by the ratio of the mass fluxes of the cometary ions and the solar wind. The distance of the contact surface is determined by the momentum balance of the cometary and the solar wind flows. These distances are in the same ratio as the velocity of the solar wind to that of the cometary particles. This factor is of the order of several hundred.

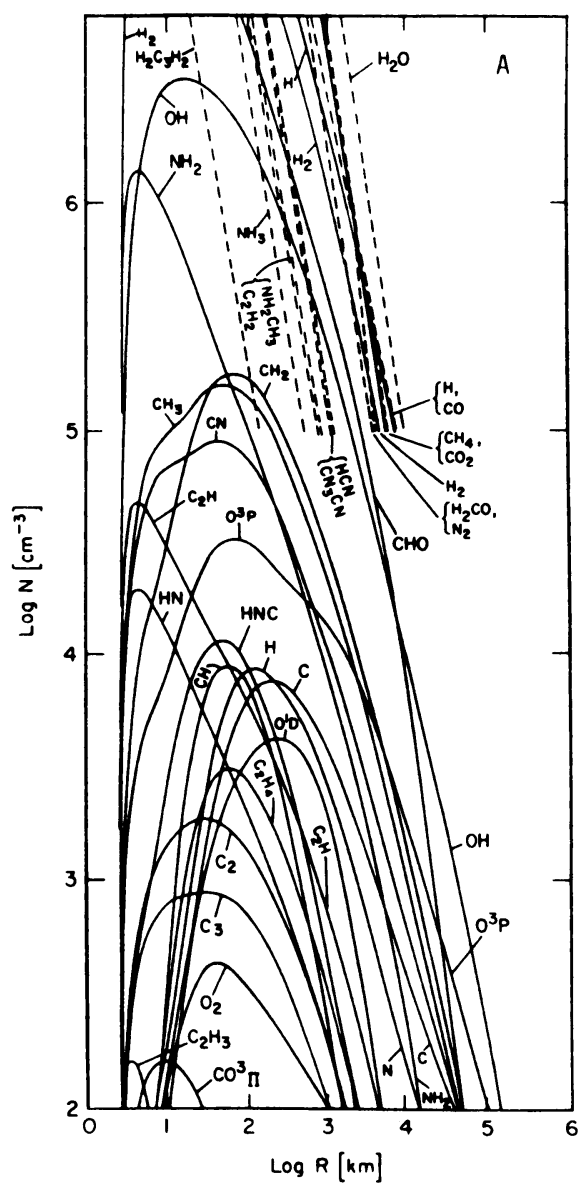

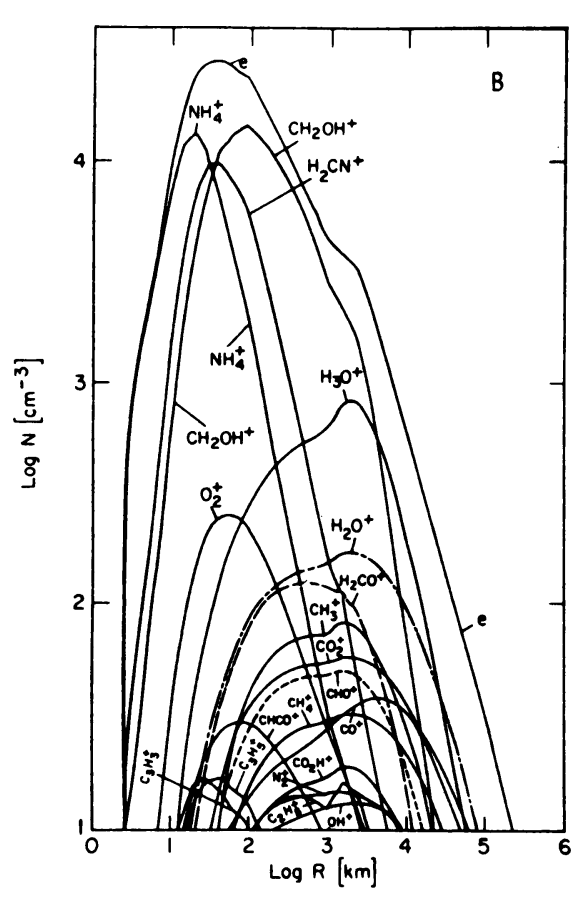

Figure 2. Number densities vs. cometocentric distance. (a) Neutral species and (b) ions, plotted separately on shifted vertical scales for clarity. 

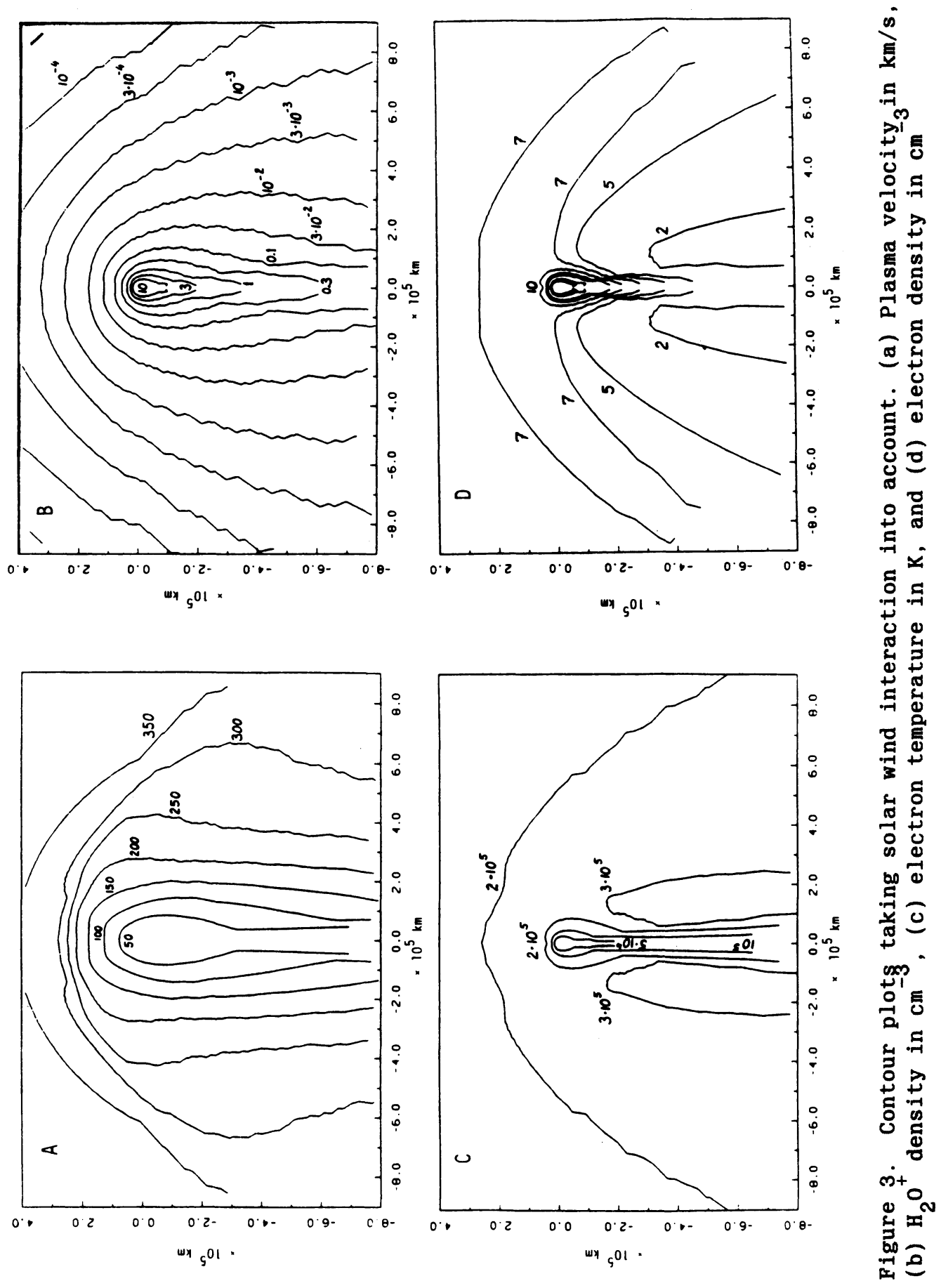
Away from the sun - comet axis the shock is oblique. Since only the normal component of the velocity is important, the shock strength decreases with increasing distance from the axis. In the shock the ion pressure is increased. As this pressure is released it accelerates the plasma (Fig. 3a) with the pickup ions (e.g., $\mathrm{H}_{2} \mathrm{O}^{+}$in $\mathrm{Fig}$. 3b) towards the tail. Other ions such as $\mathrm{CO}^{+}$show similar ${ }^{2}$ behavior. Since the flow is divergent, the densities decrease in the tail.

The shock strength is greatest on the axis. The deceleration and heating of the plasma by pickup ions continues also in an extended region behind the shock. The high pressure region formed near the axis helps to deflect the flow sideways. Nearer to the nucleus the density of the neutrals is higher and collisions of the ions with them become increasingly important. This interaction with the cold neutrals tends to decrease the velocity, temperature, and pressure of the ions.

In our model we start with a temperature of $1.9 \cdot 10^{5} \mathrm{~K}$ for the solar wind electrons. In the outer region these electrons are gradually removed by recombinations and replaced by photoelectrons. This exchange is especially efficiept in the region immediately behind the shock front. Thus the $2 \cdot 10^{5} \mathrm{~K}$ contour in Fig. 3c almost coincides with the shock front. Further inward electron impact ionization and dissociation consumes much of the energy and cools the electrons to coma temperature.

The plasma in the inner coma has the same temperature and velocity as the neutrals. The effect of the interaction of the contaminated solar wind on the expanding flow of the coma plasma starts abruptly with an inner shock, just within the contact surface that forms to separate the two flows. At some distance inside of this shock the electron temperature rises steeply and the corresponding pressure gradient decelerates the plasma and so decouples it from the flow of the neutrals. Outside of the shock the plasma is pushed subsonically around the inner coma and across the neutral gas towards the plasma tail. The transfer of momentum between neutral gas and plasma by collisions is negligible for the dense neutral gas, but is important for the plasma flow and pushes the contact surface a bit farther outward. Since both flows are subsonic, the equilibrium position is determined by the balance of the total pressure. On the inside of the contact surface the electron pressure dominates, on the outside the ion pressure dominates. The stagnation point is then determined by their balance. The ion pressure is essentially the ram pressure of the solar wind, reduced by the collisional cooling with the neutrals. This reduction of the ion pressure is quite important for the stopping of the solar wind by the electron pressure. If this were not the case, the solar wind would penetrate much farther into the inner coma, until it is stopped by the ram pressure of the radially supersonic flow of the cometary particles. The configuration depends rather sensitively on the solar wind conditions, the chemical composition, and the gas production of the comet. The magnetic field changes the situation insofar as it contributes to the total pressure on the outside of the contact surface. 
Across the contact surface the densities of all species and the pressures of the lons and the electrons can be discontinuous. Only the total pressure must be continuous. The numerical method we use at present to calculate the flow smears the contact discontinuities over several grid points and therefore obscures the discontinuities in the physical quantities.

Two-dimensional models must neglect the effects of a transverse magnetic field. The magnetic stresses in the solar wind flow on both sides of the shock discontinuity can not compete with the local kinetic energy densities. Therefore the geometry of the shock and the nearby flow of the shocked plasma are still rotationally symmetric. But, in a rotationally symmetric stagnation flow, a transverse magnetic field is piled up even if the flow is incompressible and the magnetic energy density grows proportionally to the inverse flow velocity. When near the stagnation zone the resulting magnetic stresses become comparable to the plasma pressure; they accelerate the plasma flow and divert it somewhat from rotational symmetry so that the stresses relax. These effects can be approximately incorporated in an axisymmetric model if one assumes a transverse magnetic field in the solar wind which changes its azimuth at random and faster than the convective time scale of the plasma flow in the coma.

The effect of magnetic stresses can only be described correctly by a three-dimensional model. In the existing models of this kind the chemical evolution is not calculated in detail. For expediency, the ion population is represented by a mean ion number, mass, momentum, and energy and the relevant changes by pickup processes and collisions are summed over all neutral and ion species. The results show that the magnetic field efficiently transports momentum from the ambient solar wind to the plasma tail. This increases the total stagnation pressure and consequently contracts the contact surface. Furthermore this momentum transfer contributes substantially to the acceleration of the cometary material. The field imposes an asymmetry on the flow in the tail region which tends to flatten the tail so that it becomes more concentrated near the meridional plane parallel to the interplanetary field.

\section{RESULTS AND COMPARISONS}

Column densities obtained from our models have been compared with column densities from ground based observations of several comets. For the few species for which this can be done, agreement was very good (Huebner, 1980). The average velocity of the bulk gas in our model compares well with observed values $(\approx 1 \mathrm{~km} / \mathrm{s})$ and the velocity of the fast atomic hydrogen in the outer coma of our model is in good agreement with values measured by Keller (1976) $(\approx 20 \mathrm{~km} / \mathrm{s})$.

The newest measurements for the electrons come from the ICE mission to comet Giacobini - Zinner. The measured position of the outer envelope for the solar wind interaction region is about a factor of 4 smal- 
ler when compared with the model results presented in Figs. $3 a$ to $3 d$. This is not inconsistent with our model which was based on the higher production rate of the comet in July and August and not on the lower rate found during encounter. The measured electron temperature of the coldest component at the point of closest approach to the nucleus $(\approx 7800$ $\mathrm{km})_{3}$ is 13000 to $27000 \mathrm{~K}$ and the corresponding electron density is 670 $\mathrm{cm}^{-3}$ (Meyer et al., 1985). The values from our coma model without solar wind interaction are $T_{T}=19000 \mathrm{~K}$ and $\mathrm{N}_{\mathrm{e}}=780 \mathrm{~cm}^{-3}$, and agree well with these measurements. With solar wind interaction these values are somewhat lower. Since only an upper limit of $30 \mathrm{~km} / \mathrm{s}$ can be determined for the observed electron streaming velocity at closest approach (Zwickl et al., 1985) and the final tail velocity is expected to be much higher, we conclude that the ICE intercept on 11 September 1985 occurred just at the onset of the tail.

This research is supported in part by the NASA Planetary Atmospheres Program. Work performed under the auspices of the US Department of Energy.

\section{REFERENCES}

Huebner, w. F. 1980, "Chemical Kinetics in the Coma," in Comets and the Origin of Life, Ed. C. Ponnamperuma, D. Reidel Publishing Company, Dordrecht, p. 91 .

Huebner, W. F. 1985, "The Photochemistry of Comets," in The Photochemistry of Atmospheres, Earth, the other Planets, and Comets, Ed. J. S. Levine, Academic Press Inc., New York, p. 437.

Huebner, W. F., and Keady, J. J. 1984, Astron. Astrophys. 135, 177.

Keller, H. U. 1976, Space Sci. Rev. 18, 641.

Marconi, M. L., and Mendis, D. A. 1984, Astrophys. J. 287, 445.

Meyer, N., Couturier, P., Hoang, S., Perche, C., Steinberg, J. L., Fainberg, J., and Meetre, C. 1985, EOS 66, 1016.

Schmidt, H. U., and Wegmann, R. 1982, "Plasma Flow and Magnetic Fields in Comets," in Comets, Ed. L. L. Wilkening, The University of Arizona Press, Tucson, Arizona, p. 538.

Zwickl, R. D., Baker, D. N., Bame, S. J., Feldman, W. C., Fuselier, S. A., McComas, D. J., Young, D. T., and Steinberg, J. L. 1985, E0S $66,1016$. 


\section{DISCUSSION}

P.D. FELDMAN: How much $\mathrm{CO}_{2}$ was included in the model for comet Kopff? The absence of $\mathrm{CO}_{2}{ }^{+}$in your model is inconsistent with IUE spectra of Kopff which show $\mathrm{CO}_{2}{ }^{+}$.

HUEBNER: $\mathrm{CO}_{2}$ was much less than $0.4 \%$. We could replace all the $\mathrm{CO}$ bearing molecules $\left(\mathrm{CO}, \mathrm{CO}_{2}\right.$, and $\left.\mathrm{H}_{2} \mathrm{CO}\right)$ in our assumed mixture of $\mathrm{CO}_{2}$. This should increase the $\mathrm{CO}_{2}{ }^{+}$abundance.

D'HENDECOURT: What is the reality of a comet containing $99.6 \%$ of water and how do you select the $0.4 \%$ residual with reference to other molecules?

HUEBNER: The abundance of over $99 \%$ water is based on the $\mathrm{C}_{2}$ to $\mathrm{OH}$ ratio given in the CRAF mission proposal instruction books issued by NASA. The other molecules are $\mathrm{CO}, \mathrm{CO}_{2}, \mathrm{H}_{2} \mathrm{CO}, \mathrm{NH}_{3}, \mathrm{HCN}, \mathrm{CH}_{3} \mathrm{CN}, \mathrm{C}_{2} \mathrm{H}_{2}$, $\mathrm{N}_{2}, \mathrm{H}_{2} \mathrm{C}_{3} \mathrm{H}_{2}$, and $\mathrm{CH}_{4}$ in the same proportions as we have used in other published mixtures. Only the water has been scaled up to meet NASA recommendations. I believe that the NASA recommendations for the size of the nucleus $\left(R_{O}=1.45 \mathrm{~km}\right)$ are too small.

P.D. SINGH: (i) Does your model take account of the presence of bow shock and magnetic field in coma chemistry of a comet? (ii) How efficient, in respect to other reactions, are neutral-neutral and neutral-radical reactions in a cometary atmosphere?

HUEBNER: (i) The bow shock position is determined in the twodimensional calculations. Since the bow shock is very far from the nucleus $\left(\sim 10^{5} \mathrm{~km}\right)$, the only effect on the chemistry is through charge exchange with solar wind ions, photoionization, electron impact ionization, and dissociative recombination. The magnetic field relaxation is calculated in three-dimensional model. Chemistry is considered in detail only in two-dimensional model calculations with a magnetic field that changes its azimuth randomly and faster than the convective time scale of the cometary plasma flow. (ii) Neutralneutral reactions are slow, and contribute only in very large comets in the innermost coma. Neutral molecule-radical reactions are generally also slow, but some radical-radical reactions can be fast.

GREENBERG: I like to return to this question of the amount of water that you use in comet as $99.6 \%$. I really don't understand that. The canonical value of the ratio of $O / C$ is $5: 1$ from the volatiles and by most it may go down to 2:1. This implies a large number of other molecules besides water.

HUEBNER: Comas is not $\bar{c}$ new comet. It is a periodic comet. Therefore, many of the volatiles have been depleted. However, the composition is based on the observation of $\mathrm{C}_{2} / \mathrm{OH}$ ratio.

D'HENDECOURT: What about dust? Is the UV opacity due to dust, going to be dominant in the inner coma? What could then be its influence on your model? 
HUEBNER: Dust will raise the minimum and lower the maximum in the gas temperature profile. This does not have a very large effect on the chemistry. The increase in the UV opacity will tend to delay the production of radials and ions and does reduce the chemistry somewhat, but grain surface chemistry may enhance some other reactions. Apparently, silicates are good catalysts for the production of methane and ethane.

YAMAMOTO: In your calculation of the temperature profiles in the inner coma, you reduced the effects of cooling due to expansion and of IR cooling of the $\mathrm{H}_{2} \mathrm{O}$ molecules. Which do you think is effective? HUEBNER: The expansion cooling is more effective than the radiative cooling because the IR radiation is trapped in the innermost coma. 